Scoones, Ian, Marongwe, Nelson, Mavedzenge, Blasio, Mahenehene, Jacob, Murimbarimba, Felix \& Sukume, Chrispen. - Zimbabwe's Land Reform

\title{
Dominik Kohlhagen
}

\section{(2) OpenEdition Journals}

Édition électronique

URL : http://journals.openedition.org/etudesafricaines/14500

DOI : 10.4000 /etudesafricaines. 14500

ISSN : 1777-5353

Éditeur

Éditions de l'EHESS

\section{Édition imprimée}

Date de publication : 16 septembre 2013

Pagination : 767-769

ISBN : 978-2-7132-2388-4

ISSN : 0008-0055

\section{Référence électronique}

Dominik Kohlhagen, « Scoones, lan, Marongwe, Nelson, Mavedzenge, Blasio, Mahenehene, Jacob, Murimbarimba, Felix \& Sukume, Chrispen. - Zimbabwe's Land Reform », Cahiers d'études africaines [En ligne], 211 | 2013, mis en ligne le 20 septembre 2013, consulté le 24 septembre 2020. URL : http:// journals.openedition.org/etudesafricaines/14500 ; DOI : https://doi.org/10.4000/etudesafricaines. 14500

Ce document a été généré automatiquement le 24 septembre 2020.

(c) Cahiers d'Études africaines 


\title{
Scoones, Ian, Marongwe, Nelson, Mavedzenge, Blasio, Mahenehene, Jacob, Murimbarimba, Felix \& Sukume, Chrispen. - Zimbabwe's Land Reform
}

\author{
Dominik Kohlhagen
}

\section{RÉFÉRENCE}

SCOONES, Ian, MARONGWE, Nelson, MAVEDZENGE, Blasio, MAHENEHENE, Jacob, MURIMBARIMBA, Felix \& SUKumE, Chrispen. - Zimbabwe's Land Reform. Myths \& Realities. Woodbridge, James Currey (« African Issues ») ; Sunnyside, Jacana Media, 2010, 288 p., bibl., index.

1 Plus de dix ans après le début de la réforme agraire au Zimbabwe, beaucoup d'encre a coulé au sujet des évictions violentes de fermiers blancs et l'occupation de leurs terres par des exploitants noirs au début des années 2000. Outre la violence et la désorganisation, c'est surtout le supposé échec de la réforme qui a retenu la plus grande attention dans les publications journalistiques et écrits académiques. Face à l'hyperinflation et aux pénuries alimentaires que connaît actuellement le pays, cette conclusion semblait aller de soi, bien qu aucune étude détaillée $\mathrm{n}$ ait été réalisée pour comprendre l'impact véritable de la réforme.

2 C'est cet exercice qu'entreprend l'ouvrage ci-présent. Au vu des discours dominants, le bilan qu'il propose est pour le moins surprenant. Cinq «mythes» sont annoncés en quart de couverture et dénoncés d'emblée comme erronés :

- La réforme agraire au zimbabwe aurait été un échec total ;

- Les bénéficiaires de la réforme auraient surtout été des proches du pouvoir ;

- Il n'y aurait pas eu de nouveaux investissements sur les nouvelles parcelles ; 
- L'agriculture serait en ruine, provoquant une insécurité alimentaire chronique ;

- L'économie rurale se serait effondrée.

3 Les six auteurs opposent à ces «mythes » des faits recueillis au cours d'enquêtes minutieuses effectuées dans les fermes de Masvingo, l'une des huit provinces rurales du pays. Ils dépeignent une réalité bien plus différenciée que ne le suggèrent les écrits et reportages de presse qu'ils réfutent. Chaque chapitre aborde un aspect particulier : le contexte historique et les modalités de la réforme, les profils personnels des occupants, les investissements apportés, les stratégies agraires adoptées, les rapports interpersonnels, les réseaux sociaux et économiques dans lesquels s'inscrivent les nouveaux exploitants.

4 C'est en grande partie sur la base de données chiffrées que les auteurs entreprennent leur démonstration. De nombreux tableaux récapitulatifs étayent l'analyse. Le lecteur y apprend que près de $70 \%$ des nouveaux occupants appartiennent à une catégorie que les auteurs désignent comme "gens ordinaires", sans affinité ou lien particulier avec les dignitaires de l'État ou avec des autorités locales. Contrairement aux idées reçues, seule une minorité de terres avait été attribuée à des anciens combattants, à des fonctionnaires ou à des gens d'affaires.

Des données intéressantes concernent également la productivité des parcelles redistribuées. Selon les données recueillies, près de $50 \%$ des ménages réinstallés produisent un surplus leur permettant de réinvestir des gains. Si cette réussite n'est pas identifiée par d'autres publications et sources statistiques, c'est surtout en raison des critères en fonction desquels est habituellement évaluée la production agricole. La plupart des observateurs de l'économie zimbabwéenne continuent à ne prendre en compte que les seules cultures de rente privilégiées par les fermiers évincés. Là où étaient précédemment cultivés du blé ou du tabac destinés à l'exportation, ce sont pourtant maintenant des cultures vivrières destinées à l'autoconsommation qui dominent. Ces dernières n'apparaissent souvent pas dans les statistiques.

6 Selon les auteurs, si le Zimbabwe connaît actuellement des problèmes d'autosuffisance alimentaire, ce n'est pas la réforme agraire qui est en cause. Dès le premier chapitre, les auteurs rappellent d'ailleurs que le déclin économique du Zimbabwe avait précédé de plusieurs années la réforme. Alors que les occupations de fermes avaient débuté en 2000, le dollar zimbabwéen avait commencé sa chute libre au jour du "black Friday », le 14 novembre 1997. l'origine du problème $\mathrm{n}$ avait aucun lien avec la gestion foncière : afin de pouvoir payer des primes à ces anciens combattants rentrés du Congo, le gouvernement avait fait imprimer des billets sans contre-valeur au trésor.

7 Aux images de violence et de destruction dominantes dans les reportages télévisés sur les occupations de fermes, les auteurs opposent des récits sensiblement différents. Avant même d'obtenir leurs permis d'exploitation officiels, de nombreux occupants auraient commencé à faire des "investissements impressionnants ", en construisant des abris, en défrichant les champs, en achetant des équipements et des engrais. Les auteurs estiment que les premiers investissements auraient atteint en moyenne 2000 dollars américains par exploitant. Ceci étant, ils ne précisent pas sur quel laps de temps ce montant important a été calculé, ni de quoi il est précisément composé ; le lecteur ne saura pas s'il inclut, par exemple, l'équivalent monétaire du temps de travail investi. 
8 Ce sont de telles imprécisions qui ôtent par endroits de la force à l'analyse. Les différents chapitres - dont les noms des auteurs ne sont pas précisés - sont par ailleurs de qualité très variable. Même le style d'écriture connaît des variations surprenantes. Alors que, dans certains chapitres, les auteurs s'efforcent de relever les spécificités du milieu local étudié, dans d'autres, ils tendent à généraliser leurs observations de terrain à l'ensemble du pays. Par ailleurs, les nombreuses citations et les récits reproduits dans certains chapitres ne sont pas toujours mis en contexte et apparaissent parfois davantage comme des anecdotes annexes que comme des illustrations appuyant l'argumentaire.

9 À certains égards, l'ouvrage se lit plutôt comme un recueil d'articles que comme un exposé consistant. L'ouvrage propose une grande quantité d'informations, mais ne propose pas toujours de cadre analytique pour les rassembler. À de nombreux endroits, il évoque ainsi des dynamiques sociales qui auraient mérité d'être mises en lien: la redéfinition de statuts et d'attributs d'autorité, des changements dans les rapports de genre, l'émergence de nouvelles formes de socialité locales ou de pratiques mystiques, pour n'en citer que certaines. C'est peut-être parce qu'ils entendaient avant tout répondre aux critiques que rencontre la réforme à l'échelle macroscopique que les auteurs ont quelque peu négligé la richesse de leurs observations recueillies à l'échelle locale. À ce sujet, il serait vivement souhaitable que d'autres recherches prolongent les pistes très intéressantes lancées par l'ouvrage.

10 En tout état de cause, le livre ci-présent est assurément incontournable pour comprendre les conséquences de la réforme agraire zimbabwéenne. De manière plus générale, il livre aussi une démonstration intéressante des avantages de l'agriculture familiale de subsistance face à l'exploitation commerciale à plus grande échelle. Sans doute pourra-t-on reprocher aux auteurs de ne pas renseigner le lecteur sur le sort des dizaines de milliers d'employés agricoles qui ont perdu leur travail. Aussi semble-t-il difficile de se laisser convaincre du succès de la réforme alors que, au-delà, le pays vit une période de violence et de misère sans précédent. Mais grâce à cette publication, le débat scientifique est enfin ouvert. 